\title{
Prototype of Instrument for Minimally Invasive Surgery with 6-Axis Force Sensing Capability
}

\author{
Ulrich Seibold, Bernhard Kübler, and Gerd Hirzinger \\ Institute of Robotics and Mechatronics \\ German Space Center \\ Münchnerstraße 20, 82234 Wessling, Germany \\ ulrich.seibold@dlr.de
}

\begin{abstract}
Minimally invasive surgery (MIS) challenges the surgeon's skills due to his separation from the operation area which can only be reached with long instruments. To overcome these drawbacks, minimally invasive robotic surgery (MIRS) plays an important role. This paper describes the development of actuated and sensorized instruments for minimally invasive robotic surgery which help to increase the surgeon's immersion and dexterity.
\end{abstract}

Index Terms - Minimally Invasive Surgery, Articulated Grasper, Force-Torque Sensor, Kinesthetic Feedback, Sterilization.

\section{INTRODUCTION}

\section{A. Minimally Invasive Surgery (MIS)}

Minimally invasive surgery is an operation technique established in the 1980s. The surgeon operates with specially designed surgical tools through a small incision of typically only $1 \mathrm{~cm}$ on the patient's skin. The patient benefits from a reduction of surgical trauma to tissues, decreased pain and significantly shorter hospitalization. Cosmetic benefits due to smaller visible scars must also be mentioned. These advantages for the patient however are accompanied by significant disadvantages for the surgeon compared to open surgery. The direct hand-eye coordination present in open surgery is lost [1], as is the direct manual access to the operation site. The long instruments are moved about the fixed point of incision, therefore two degrees of freedom (DoF) are tied and a loss of dexterity inside the patient's body results [2], [3], [4]. The movement is also subject to scaling depending on the depth of insertion. These are significant drawbacks of MIS, which make complex tasks like knot tying very time consuming and require intensive training. As a consequence, MIS did not prevail as desired by patients and surgeons. Only cholecystectomies (gallbladder removals) are performed in $95 \%$ or more cases using minimally invasive procedures.

\section{B. Minimally Invasive Robotic Surgery (MIRS)}

Key technologies to overcome the drawbacks of manual MIS are robotic and mechatronic systems, which help the surgeon to regain virtually direct access to the operating field. These technologies applied to minimally invasive surgery in combination with telepresence and telemanipulation approaches lead to minimally invasive robotic surgery: In a MIRS setup the instruments are not directly manipulated anymore. Instead, they are remotely commanded by the surgeon who comfortably works in front of a console while the instruments themselves are moved by specialized robotic arms [5], [6].

MIRS telepresence systems help the surgeon to overcome barriers, such as the patient's chest or abdominal wall, which separate him from the operating area and cause the drawbacks mentioned before: With appropriate control algorithms the undesired reverse hand motion can be avoided and the direct hand eye coordination is reestablished [7]. The desired downscaling and removal of tremor of the surgeon's hand motion before being transmitted to the robot is another benefit: movements of instruments become more accurate than in open surgery. Actuated instruments with two additional DoFs facilitate full dexterity inside the human body allowing every point in the work space to be reached with arbitrary orientation. Furthermore, MIRS systems allow for the realization of autonomous functions (such as motion compensation of the beating heart [8]) and enable surgeons to perform new operation techniques like endoscopic minimally invasive bypass surgery at the beating heart [9]. Until now mainly two commercial MIRS systems are available and also in clinical use: the ZEUS system from Computer Motion Inc. [6] and the daVINCI system from Intuitive Surgical Inc. [10] Recently, both companies have announced their merger agreement.

\section{Haptics in Minimally Invasive Robotic Surgery}

Friction generated within the instrument and between the instrument and the access port greatly exceeds manipulation forces at the operation site (which are in the range of $0.3 \mathrm{~N}$ for bypass grafting [11]), hindering haptic feedback. Palpation of tissue is not possible as there is no direct access to the operating field. Surgeons are able, through experience, to interpret tissue deformation as a measure of external forces and thus compensate for the lack of haptic information. Unfortunately, tissue properties depend on the patient and may also vary with time [12]. This visual compensation is feasible only with elastic materials as bone structures and suture materials do not allow for visual 
judgment due to their high stiffness.

A design for an experimental hand held force feedback instrument which measures and displays the grasping force during palpation was proposed by $\mathrm{J}$. Rosen [13]. Experiments comparing the tissue recognition accuracy palpating by hand versus using conventional MIS instruments showed the performance of the force feedback instrument being close to that of an ungloved hand. The effect of haptic feedback on the performance of surgeons has been studied by R. Wagner [12]. In this laboratory setup a commercial six DoF force-torque sensor (FTS) was located in the shaft of an instrument, close to the handle. No significant influence on precision and speed of a dissection procedure was found, however the rate of errors and the applied average force was greatly reduced. It is expected that miniaturized force sensors allow for the accurate measurement of contact forces between tissue and instrument. This information can, in combination with kinesthetic feedback devices and appropriate control laws, help to reduce unintentional damage to tissue and suture material. Although direct haptic feedback is a prospective improvement to MIRS, it is currently not available in systems for minimal invasive surgery.

In Section II-A the design of a distal force-torque sensor is presented. The sensor is miniaturized (outer diameter $10 \mathrm{~mm}$ ) in order to fit through a standard trocar. Section II-B describes the development of an actuated pair of forceps. The actuation is realized by cables whereas the cable alignment is selected such that - in contrast to regular universal joint - a nearly linear transmission behavior is achieved. Section II-C gives an overview of the developed propulsion unit which consists of two parts. The distal part can be steamsterilized in a digester, and the proximal part (the actual propulsion unit) contains the electro-mechanical and electronic components. The propulsion unit also incorporates force and position sensors which will be used to increase the positioning accuracy and enable the decoupling of manipulation from grasping forces. Finally, Section III gives an overview of initial functional tests of the sensor and proposed functional tests of the completed instrument.

\section{Sensorized, Articulated Grasping INSTRUMENT}

Commonly used during minimally invasive surgery are various tools with gripper-like end-effectors (forceps, scissors, clip applicators). Instead of a handle in telerobotic surgery an electromechanical propulsion unit is located at the proximal end, while the distal end contains an additional 2 DoF joint (Fig. 1). The functional tool-tip is actuated by the propulsion unit with cables or push/pull rod. Grouping this instrument in functional blocks, the following components can be distinguished:

- The force-torque sensor measuring manipulation forces at the instrument's tip.

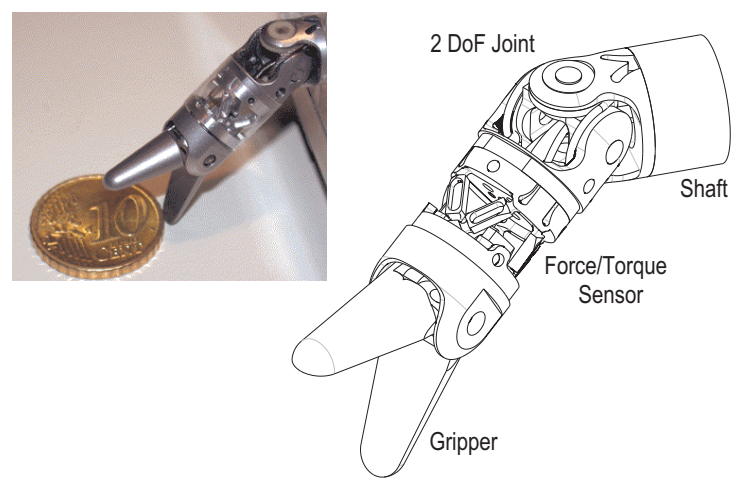

Fig. 1. DLR instrument for minimally invasive surgery.

- The mechanical joint/gripper assembly actuated by cables.

- (Not shown in Fig. 1), the drive assembly actuating the joint and separated thereof by the shaft. It also provides the electrical and mechanical interface between robot and instrument.

\section{A. Distal Force-Torque Sensor}

Precise force information can only be obtained inside the patient's body close to the operation site, minimizing the errors due to friction between the instrument and the point of incision. The sensor should be separated from the drive mechanism to prevent the influence of backlash and actuation forces on the sensor's performance. Currently no suitable commercial force sensor is available for the integration in the shaft of a surgical instrument. Placement of a FTS was considered in three different locations of the instrument tip [14]:

- In the jaws of the gripper. The jaws issue the most severe size constraints. Additionally, coupling between manipulation and grasping forces occurs, and measured loads depend on the jaw angle. A great number of instruments in MIS differ only in the shape and function of the jaws. Integration of the sensor would undermine adaptability of the instrument design.

- In the shaft proximal to the joint. This placement does not require the electrical connection to be routed through the joint, which will prolong the life expectancy of sensor and electrical connections. However, three sets of drive cables for the joint and gripper need to be routed through the sensor and manipulation forces have to be corrected for the joint angle. Furthermore, the sensor is subjected to the actuation forces including cable tension which greatly exceed the manipulation loads. The measurement range of the sensor must accommodate for the actuation forces, thereby greatly reducing the sensitivity to the manipulation reactions. Compensation is highly difficult since knowledge of all five cable forces at the sensor location is required 
- Between gripper and joint. At this location the sensor is only subjected to the gripper cable force which is acting centrically. Since this force is measured for the calculation of the gripping force, the FTS output can be compensated for simultaneously. However, the electrical connection to the sensor has to be routed through the joint, requiring highly flexible, isolated, multistrand wires. This location requires the sensor to be of roughly cylindrical shape, with a preferably central hollow section to accommodate for the gripper drive cable and mechanics.

A force-torque transducer based on the Stewart Platform is well suited for this application. Advantages include high stiffness, adaptable properties, annular shape and scalability. Furthermore, only linear force transducers are required which can be placed on a hexagonal cross-section (Fig. 4). This placement facilitates the use of measurement systems other than foil-backed strain gauges, e.g. thin-film grids. Analysis and properties of Stewart Platform transducers were presented by D.R. Kerr [15] and M. Sorli [16], the latter outlining a set of variables $(R, L, \alpha, \beta, \gamma$, shown in Fig. 2) sufficiently describing the geometry and thus the properties of the sensor.

The characteristic matrix $\mathbf{A} \in \mathbb{R}^{6 \times 6}$ describing the transformation of link forces to externally applied loads

$$
\begin{gathered}
{\left[F_{x}, F_{y}, F_{z}, M_{x}, M_{y}, M_{z}\right]^{T}=} \\
\mathbf{A} \cdot\left[F_{1}, F_{2}, F_{3}, F_{4}, F_{5}, F_{6}\right]^{T},
\end{gathered}
$$

is calculated using the method described by [16].

$$
\begin{aligned}
& \mathbf{A}=-\frac{1}{2} \text {. } \\
& {\left[\begin{array}{ccc}
-2 n & 2 n & \sqrt{3} m+n \\
-2 m & -2 m & m-\sqrt{3} n \\
-2 q & -2 q & -2 q \\
2 a q & 2 a q & -a q \\
0 & 0 & a q \sqrt{3} \\
-2 a n & 2 a n & -2 a n
\end{array}\right.} \\
& m=\cos (\alpha) \cos (\beta) \\
& n=\cos (\alpha) \sin (\beta) \\
& q=\sin (\alpha)
\end{aligned}
$$$$
\begin{array}{cc}
\sqrt{3} m-n & -\sqrt{3} m+n \\
m+\sqrt{3} n & m+\sqrt{3} n \\
-2 q & -2 q \\
-a q & -a q \\
a q \sqrt{3} & -a q \sqrt{3} \\
2 a n & -2 a n
\end{array}
$$$$
\left.\begin{array}{c}
-\sqrt{3} m-n \\
m-\sqrt{3} n \\
-2 q \\
-a q \\
-a q \sqrt{3} \\
2 a n
\end{array}\right]
$$

To find a sensor geometry that is well conditioned and optimized for the force range expected in a surgical application, the following optimization method is used. The radius of the base $R$ and the link length $L$ are determined by the space available in the instrument. For geometrically valid combinations (nonintersecting links) of $R, L, \alpha, \beta, \gamma$ are used to calculate A. Inverting $\mathbf{A}$ yields the transformation of externally applied loads to link forces $\mathbf{J} \in \mathbb{R}^{6 \times 6}$. Selecting various sets of maximally expected external loads $\left[F_{x}, F_{y}, F_{z}, M_{x}, M_{y}, M_{z}\right]^{T}$ are selected. They must at least contain loads in the 6 principal directions. Every member of the load set is pre-multiplied by $\mathbf{J}$, yielding the corresponding set of internal leg forces

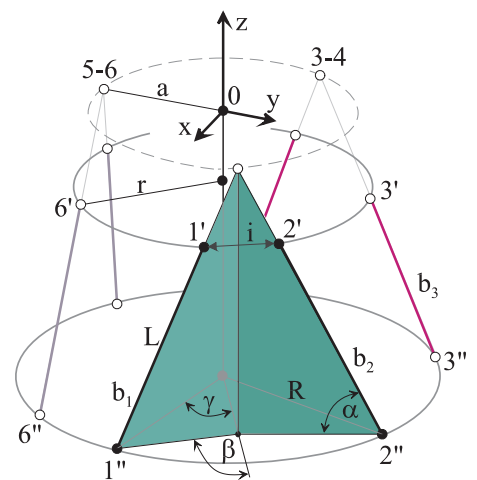

Fig. 2. Geometrical parameters of Stewart Platform: Base radius $R$; platform radius $r$; radius of link intersection $a$; link length $L$; joint separation at platform $i$; Lower joints 1 "-6"; upper joints 1'-6'; link intersections 1-2, 3-4, 5-6, and links $b_{1}-b_{6}$.

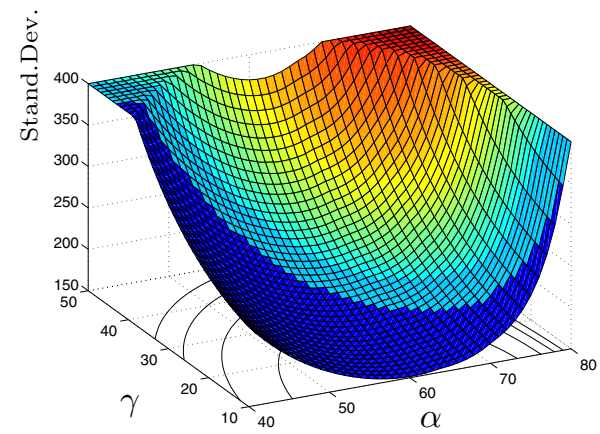

Fig. 3. Standard deviation for valid geometrical parameters. Colors denote solutions with equal leg separation $i$ at the platform.

$\left[F_{1}, F_{2}, F_{3}, F_{4}, F_{5}, F_{6}\right]^{T}$. The standard deviation $s$ of the internal leg force set (denoted in Fig. 3) is a measure of the isotropy of the sensor structure with respect to the external load set. This however is not an isotropy in the classical definition, since the external loads in the principal directions need not be equal. For the load set $F_{x, y, z}=30 \mathrm{~N}$, $M_{x, y}=300 \mathrm{Nmm}, M_{z}=150 \mathrm{Nmm}$ the following parameters were selected as optimal sensor geometry: $R=4.2 \mathrm{~mm}, L=3.9 \mathrm{~mm}, \alpha=57^{\circ}, \beta=90^{\circ}, \gamma=36^{\circ}$, yielding a standard deviation of $s=236$ and a joint separation of $i=1.1 \mathrm{~mm}$. Given appropriate design of flexural hinges and leg cross-section, the results of an FEM analysis are in very good agreement with the prediction by the ideal analytical model [14]. Fig. 4 shows the surface strain for an external load of $30 \mathrm{~N}$.

\section{B. Joint and Gripper Mechanics}

Based on the proposed placement of the force-torque sensor, joint mechanics where the last axis of rotation coincides with the axis of the gripper (Endo-Wrist of Intuitive Surgical Inc. [10]), can hardly be used.

The length of the joint assembly during surgery is restricted by manipulability considerations and the dis- 


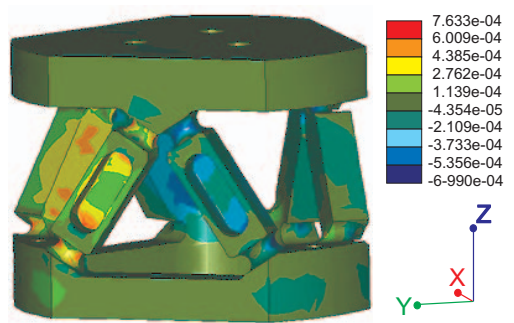

Fig. 4. FEM Simulation of average strain in flexural jointed Stewart Platform using the following parameters: $R=4.2 \mathrm{~mm}, L=3.9 \mathrm{~mm}, \alpha=57^{\circ}, \beta=90^{\circ}, \gamma=36^{\circ}$,

$F_{y}=30 \mathrm{~N}$.

tance between skin and operation site. For abdominal procedures this distance can be increased by insufflation, however due to the rigidity of the ribcage this is not an option with heart procedures. The joint and gripper mechanics should therefore be kept as short as possible. The articulated joint shown in Fig. 1 and Fig. 5 closely resembles a universal joint with intersecting axes, actuated by cable drives. This design allows for twisting the gripper about its longitudinal axis without pivoting the instrument shaft about the point of insertion. The range of motion in the joint is restricted to about $\pm 40^{\circ}$ in both directions, caused by an inherent limitation of universal joints. However it is possible to pair two universal joints to achieve a range of motion of $\pm 80^{\circ}$. The setup of the drive cables in the joint forces the cables to run tangent to the drive pulleys at all times. Therefore the lengths of both cable loops remain constant for every joint position. The middle of each cable loop is tied to the distal component of the joint (see Fig. 5), while the proximal ends are connected crosswise at the actuators. With this layout only two fixed rotary drives are needed to fully actuate the joint, yielding linear transmission characteristics. Driving only one actuator results in a tilting motion of the instrument tip at $45^{\circ}$ angle to the principal axes of the joint (see Eq. 2):

$$
\begin{aligned}
\theta_{8} & =\frac{r_{M}}{2 r_{a}}(\beta-\alpha), \\
\theta_{9} & =\frac{r_{M}}{2 r_{a}}(\beta+\alpha)
\end{aligned}
$$

with $r_{M}$ : radius of motor pulley, $r_{a}$ : radius of joint pulley $(3 \mathrm{~mm}), \alpha, \beta$ : joint angles and $\theta_{1}, \theta_{2}$ : actuator positions.

Fig. 5 shows a simplified model of the joint and cable mechanics. $J_{2}$ is the lumped moment of inertia for the actuator seen from the output, $D_{2}$ accounts for friction on the actuator side. $K$ is the lumped elasticity of each cable segment. $M$ is the mass of FTS and gripper with the distance $l$ from the center of mass to the joint axis. However $M$ can be neglected for actuation frequencies up to $4 \mathrm{~Hz}$. The parameter $D_{2}$ combines friction in the joint (depending on the environmental conditions wet/dry), stick-slip effects and losses due to pulleys.

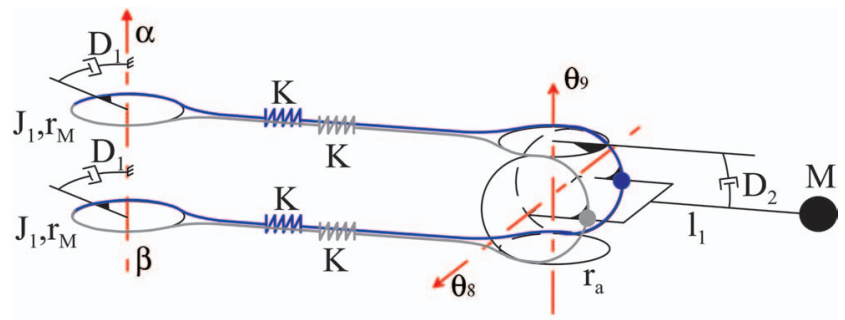

Fig. 5. Articulated joint with drive cables. Cables are fixed to the distal joint component (right) and connected crosswise at the drive (left).

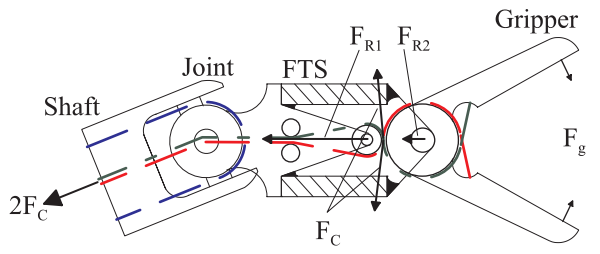

Fig. 6. Decoupling of gripper actuation force from 6DoF FTS, with gripping force $F_{g}$, cable actuation force $F_{c}$, resulting force acting on the base of the FTS $F_{R 1}$ and resulting force 'seen' by the FTS $F_{R 1}$ (forces not to scale).

The possible range of values for this parameter will have to be determined in a series of experiments.

Maximum cable force for the joint actuation is $100 \mathrm{~N}$. To guarantee zero backlash, the cables are prestressed with the maximally expected driving force, accounting for a worst case cable force of 200 N. Resulting manipulation forces are $20 \mathrm{~N}$ at the instrument tip, with the gripping force being $20 \mathrm{~N}$ respectively.

The gripper is actuated by one cable counteracted by a spring. The cable force necessary to close the gripper and securely hold a needle is estimated to be $70 \mathrm{~N}$. As described in Section II-A this actuation force is greatly exceeding the measurement range of the FTS. Fig. 6 shows a mechanical arrangement for supporting most of this actuation force $\left(F_{R 1}\right)$ at the base of the sensor. The cables for actuating the gripper are routed over pulleys attached to the base. This generates two opposing force components $\left(F_{c}\right)$ between base and platform of the sensor which are essentially orthogonal to the axis of the sensor and therefore cancel each other except for a small residual. The cables are slightly offset thereby generating a small torsional moment and a small axial force $\left(F_{R 2}\right)$ which are measured by the FTS. These can be compensated for in the output signal as the actuation force for the gripper and the cable geometry are known.

\section{Propulsion Unit}

This unit provides actuation for the distally located joint and gripper and signal conditioning for the distal force-torque sensor. The unit is connected to the tool center point (TCP) of a medical robot providing mechanical, electrical and data interfaces. Within the 
development of a MIRS propulsion unit the following essential topics have to be considered:

- The components with patient contact have to be sterilizable for multiple, cost effective use.

- For adequate position and force control (e.g. for motion compensation) a mechanical transmission free of backlash is required. Additionally actuation forces and actuator positions should be measured.

- The propulsion unit should be as light and compact as possible so as not to influence the dynamics of the surgical robot negatively and to avoid collisions with other instruments.

The sterilization requirement is solved by separating the unit into two reconnectable components: one steam-sterilizable section in patient contact without any electro-mechanical or thermally-instable components and one spray-sterilizable section not in patient contact containing all thermally-instable components (motors and electronics, see Fig. 7).

Since effective motion control is impossible in a mechanical system afflicted with backlash, the interface between these two components has to be free of any backlash. This is accomplished by adjusting the power transmitting surfaces during initial assembly of the unit, compensating for manufacturing tolerances. Thereafter the separation and reconnection must be easily accomplished for health personnel under operating room conditions. During clinical use separation and reconnection is accomplished with a spring loaded latch mechanism and lateral joining motion (see Fig. 7).

Using highly integrated mechatronic design the 3 DoF propulsion unit could be accommodated in a cylindrical shape of $65 \mathrm{~mm}$ in diameter and a height of $85 \mathrm{~mm}$, nevertheless generating cable forces of $100 \mathrm{~N}$ (cable tension is generated independently of the actuators) and an actuator frequency of $4 \mathrm{~Hz}$. These dimensions include the necessary electronic components (power electronics, control logic and sensor evaluation electronics).

Because of the high force density, cables were chosen as force transmission media. Steel cables (material 1.4401) were used in a previous design. Although having a higher Young's Modulus $\left(E=2.1 \cdot 10^{5} \frac{\mathrm{N}}{\mathrm{mm}^{2}}\right.$ for steel compared to $E=6.5 \cdot 10^{4} \frac{\mathrm{N}}{\mathrm{mm}^{2}}$ for Vectran $\left.{ }^{\circledR}[17]\right)$, they exhibit significant creep under prolonged tension. Synthetic fibre cables seemed to be favorable: a 12-time plaited high module polyester material (trade name: Vectran ${ }^{\circledR}$ ) with a diameter of $0.6 \mathrm{~mm}$ presents much lower flexural rigidity in comparison to commercially available steel cables, providing a smaller bending radius. Furthermore Vectran ${ }^{\circledR}$ is stable against acids and bases commonly used for sterilization, withstands a temperature up to $330^{\circ} \mathrm{C}$ and does not collect moisture or ichor. Because of the initial loading of the cables it is also important that Vectran ${ }^{\circledR}$ has no incline to creep.

Integrated in the propulsion unit are actuator position and torque sensors. By comparing this information
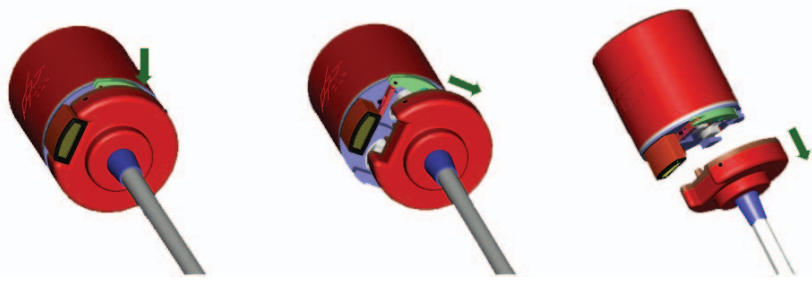

Fig. 7. Propulsion unit. Procedure to deconnect the two components of the unit.

to the data collected by the distal FTS it is possible to extract information about cable forces and the friction in the drive chain (generated in the joint, shaft and propulsion unit). As no distal position sensors are present, the actual position of the instrument tip is not precisely known. Deviation from the desired position arises from elastic cable elongation caused by external manipulation forces. The redundancy of sensors permits the compensation of these position errors and also allows plausibility checks. It thereby serves as a safety mechanism against cable tear or dislocation from the pulleys.

\section{ExPERIMENTAL Results}

Initially the components of the instrument are tested separately. The sensor is calibrated using external loads of $F_{x, y, z}=2 \mathrm{~N}$ and $M_{x, y, z}=80 \mathrm{Nmm}$ consisting of weights attached by strings and pulleys. Multi-point calibration using a least-square optimization is planned for the future. For the calibration every measurement is averaged over 500 samples to account for noise in the sensor signal.

Subsequent to calibration, the sensor was loaded at $0.25 \mathrm{~N}$ increments to a maximal load of $\pm 2.5 \mathrm{~N}$ in all primary force directions to determine the quality of the calibration as well as linearity of the sensor. Fig. 8 shows the results of a consecutive loading-unloading cycle in each of the three primary force directions. Gain deviation of the $y$-direction is attributed to non-decayed temperature drift during calibration (refer to Fig. 4 regarding the orientation of the coordinate system). No hysteresis is visible, however forces in y-direction show slight nonlinearity. In Fig. 9 some crosstalk between lateral and axial load directions is evident; an external force only acting in the $\mathrm{y}$-direction produces a small response in the $\mathrm{x}$ - and $\mathrm{z}$ - direction. The crosstalk between the two lateral load directions in Fig. 9 is caused by a slight rotational misalignment of the instrument during the experiment. The z-component is mainly caused by parasitic bending of links due to the flexural hinges which transmit not only forces axial to the link, but also bending moments. These are recorded by the strain gauges which are located slightly off the neutral bending plane. The force resolution of the sensor is $0.25 \mathrm{~N}$ in z-direction and $0.05 \mathrm{~N}$ in $\mathrm{x}$ - and y-directions. Currently the drive and joint mechanics are tested 


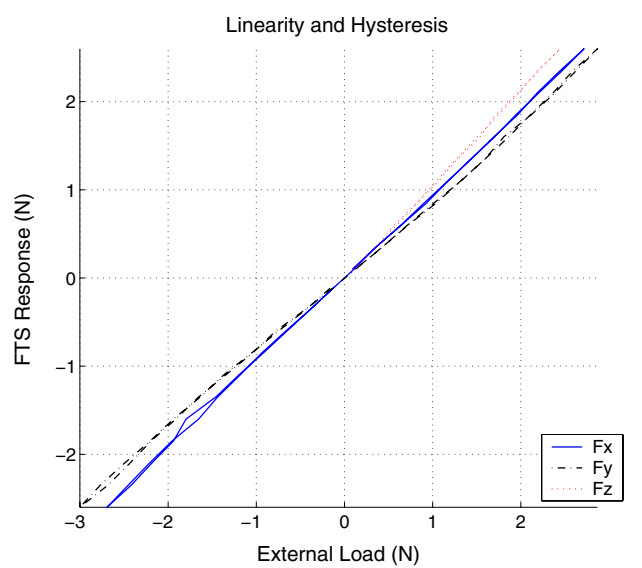

Fig. 8. Preliminary response of FTS to externally applied loads.

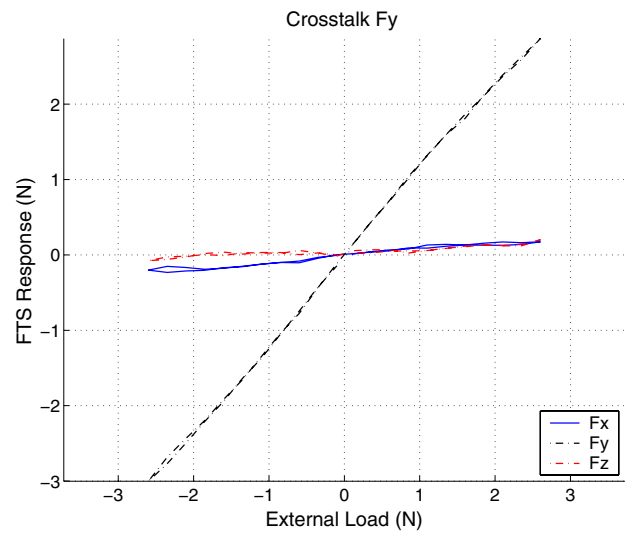

Fig. 9. Crosstalk for externally applied load in y-direction.

in order to identify the mechanical parameters of drive and joint kinematics. The propulsion unit is capable of generating manipulation and gripping forces of $20 \mathrm{~N}$ at the instrument tip with joint speeds up to $4 \frac{\mathrm{rad}}{\mathrm{s}}$. After a position controller has been implemented, the tracking accuracy will be evaluated. The performance of the propulsion unit is high enough to follow the trajectory of a natural landmark on the heart surface. The trajectory is extracted from a video sequence recorded during a beating heart coronary artery bypass grafting (CABG) procedure [7]. Further experimental results will be available shortly.

\section{Conclusion}

Tele-surgical MIRS systems are set out to broaden the application fields of MIS and to improve the quality of surgical interventions. They should provide a high level of immersion to the surgeon into the remotely performed operation: The surgeon regains direct virtual access to the operating field.

A novel design for a generic articulated instrument is presented. Actuated instruments are necessary to provide full dexterity inside the patient. This makes MIS more similar to open surgery and it is to be expected that more operations will be carried out in a less invasive way. The feasibility of integrating a miniaturized force-torque sensor at the instrument's tip was demonstrated. In combination with appropriate input devices and control structures this sensor can provide realistic kinesthetic feedback of the remote forces. This gives the surgeon direct access to manipulation forces inside the patient and allows for a more delicate manipulation of tissue, avoiding unintentional damage.

\section{REFERENCES}

[1] M. R. Treat, Computer-Integrated Surgery. MIT Press, 1995, ch. A Surgeon's Perspective on the Difficulties of Laparoscopic Surgery, pp. 559-560.

[2] M. Helmy, "A comparative study between laparoscopic versus open appendicectomy in men," J Egypt Soc Parasitol, Aug. 2001.

[3] M. Huang, P. Wei, C. Wu, R. Chen, and W. Lee, "Needlescopic, laparoscopic, and open appendectomy: a comparative study," Surg Laparosc Endosc Percutan Tech., Oct. 2001.

[4] S. Sauerland, R. Lefering, and E. Neugebauer, "Laparoscopic versus open surgery for suspected appendicitis," Cochrane Database Syst Rev, 2002.

[5] T. Ortmaier, U. Seibold, U. Hagn, D. Boehm, H. Reichenspurner, and G. Hirzinger, "Autonomy and haptic feedback in minimally invasive robotic surgery," Fourth Annual Meeting of the International Society for Minimally Invasive Cardiac Surgery (ISMICS), Munich Germany, 2001.

[6] J. M. Sackier and Y. Wang, Computer-Integrated Surgery. MIT Press, 1995, ch. Robotically Assisted Laparoscopic Surgery: From Concept to Development, pp. 577-580.

[7] T. Ortmaier, Motion Compensation in Minimally Invasive Robotic Surgery. VDI Verlag, 2003, phD Thesis.

[8] Y. Nakamura, "Virtual stillness and small size robot system that occupies less space in OR," in International Conference on Robotics and Automation (ICRA) 2003, Workshop Recent Advances in Medical Robotics, Taipei, Taiwan, 2003.

[9] V. Falk, A. Diegeler, T. Walther, N. Löscher, B. Vogel, C. Ulmann, T. Rauch, and F. W. Mohr, "Endoscopic coronary artery bypass grafting on the beating heart using a computer enhanced telemanipulation system," Heart Surg Forum, vol. 2, pp. 199-205, 1999.

[10] G. Guthart and J. Salisbury, "The intuitive telesurgery system: Overview and application," in Proceedings of the 2000 IEEE International Conference on Robotics and Automation, 2000.

[11] D. Salle, P. Bidaud, and G. Morel, "Optimal design of high dexterity modular MIS instrument for coronary artery bypass grafting," in ICRA, 2004.

[12] C. Wagner, N. Stylopoulos, and R. Howe, "The role of force feedback in surgery: Analysis of blunt dissection," in Proc. of the 10th Annual Haptics Symposium, March 2002.

[13] J. Rosen, M. MacFarlane, C. Richards, B. Hannaford, and M. Sinanan, "Surgeon-tool force/torque signatures - evaluation of surgical skills in minimally invasive surgery," in Medicine Meets Virtual Reality, San Francisco, CA, vol. 7, January 1999.

[14] U. Seibold and G. Hirzinger, "A 6-axis force/torque sensor design for haptic feedback in minimally invasive robotic surgery," in MICRO.tec 2003 2nd VDE World Microtechnologies Congress, 2003.

[15] D. Kerr, "Analysis, properties and design of a stewartplatform transducer," Journal of Mechanisms, Transmissions and Automation Design, vol. 111, no. 25, march 1989.

[16] M. Sorli and S. Pastorelli, "Six-axis reticulated structure force/torque sensor with adaptable performances," Mechatronics, vol. 5, no. 6, pp. 585-601, 1995.

[17] B. Kübler, "Entwicklung einer integrierten Antriebseinheit für ein minimal invasives Zangeninstrument in der robotergestützten Chirurgie," Master's thesis, Universität Stuttgart, April 2003. 